\title{
Evolutionary Strategy for Political Districting Problem Using Genetic Algorithm
}

\author{
Chung-I Chou ${ }^{1}$, You-ling $\mathrm{Chu}^{1}$, and Sai-Ping $\mathrm{Li}^{2}$ \\ ${ }^{1}$ Department of Physics, Chinese Culture University, Taipei, Taiwan 111, R.O.C. \\ ${ }^{2}$ Institute of Physics, Academia Sinica, Taipei, Taiwan 115, R.O.C.
}

\begin{abstract}
The aim of the Political Districting Problem is to partition a zone into electoral districts with constraints such as contiguity, population equality, etc. By using statistical physics methods, the problem can be mapped onto a $q$-state Potts model system, and the political constraints are written as an energy function with interactions between sites or external fields acting on the system. This problem is then transformed into an optimization problem. In this paper, we apply the genetic algorithm to Political Districting Problem. We will illustrate the evolutionary strategy for GA and compare with results from other optimization algorithms.
\end{abstract}

Keywords: Genetic Algorithm, $q$-state Potts model, Political Districting Problem.

\section{Introduction}

The aim of the Political Districting Problem is to partition a territory into electoral districts subject to constraints such as contiguity, population equality, etc. In our previous work [1, we have mapped the political districting problem onto a $q$-state Potts model In this model, we use "precinct" as the smallest unit and identify it as a site. The constraints can then be written as interactions between sites or external fields acting on the system. Districting into $q$ voter districts is thus equivalent to finding the ground state of this $q$-state Potts model which then becomes an optimization problem. In this earlier work, we used simulated annealing method to study both computer generated and real world districting cases.

Since genetic algorithm (GA) is known to be very useful in studying optimization problems, we will here apply this method to the Political Districting Problem. There are indeed difficulties in applying GA to this problem. The usual GA maps variables to some 1D gene code, and to generate these gene codes (or chromosomes) in a gene pool as the parent generation. Evolutionary operators such as crossover, mutation, selection, will help to generate the child generation. As the evolution goes on, chromosomes with better fit will appear. For a spin system like we have here, problems will appear in the crossover stage. The usual crossover is to swap some part of the sequences among 1D parent gene codes. Since the Political Districting Problem is a 2D problem, only randomly 
swapping the 1D parent gene codes will lose the important 2D structural information. Most of the newly generated chromosomes will then behave worse than their parents. The crossover strategy for the Political Districting Problem thus needs to be modified. We here design a process to mix the boundaries of the districting zone of a pair of parents, and to generate a child from these mixed boundaries. In this way, each child will in some way look similar to its parents. In the following, we will illustrate our evolutionary strategy for GA and compare the results with other optimization algorithms.

\section{Model for Political Districting Problem}

In our model [1], the total number of sites (precincts) is equal to $N$ and each spin can have $q$-states (voter districts). Each spin can have its state function as $S_{i}=$ $1 \ldots q$. The goal is to find the ground state of this $q$-state Potts model with the interaction given by the constraints. We consider population equality, contiguity and compactness here, which are the most common constraints people consider.

For population equality, we associate a random field to the site (precinct) $p_{i}$. It is easy to see that when $p_{i}$ is a constant for every $i$, the population for each site (precinct) is equal. Therefore, the magnetization (the total voter population) of a voter district $P_{l}$ can be written as $P_{l}=\sum_{i=1}^{N} p_{i} \cdot \delta_{S_{i}, l}$, where $\delta_{i, j}$ equals to 1 when $i$ and $j$ are equal and zero otherwise, the total population is $P_{0}=\sum_{l=1}^{q} P_{l}$, and the average population for each voter district is $\langle P\rangle=P_{0} / q$. Hence we can write the average population for each voter district as $E_{P}=\sum_{l=1}^{q}\left|1-\frac{P_{l}}{\langle P\rangle}\right|$. The smaller this energy is, the closer it is to the average value.

We next consider the constraint of contiguity and compactness. Define a connection table for each spin as $C_{i, j}$ which equals to 1 when spins $i$ and $j$ are connected to each other and zero otherwise. We here define the boundary of the domain of spins as $E_{D}=\sum_{i, j}\left(1-\delta_{S_{i}, S_{j}}\right) \cdot C_{i, j}$, When this function is in its minimum, the district will have the smallest number of precincts on its boundary. The total energy is therefore given by $E=\lambda_{P} E_{P}+\lambda_{D} E_{D}$. Varying $\lambda$ will affect the contribution of each constraint to the total energy function.

With this energy function, the problem now becomes an optimization problem and can be solved by using optimization algorithms.

\section{Strategy for Genetic Algorithm}

As mentioned above, genetic algorithms try to simulate the evolutional process of biological system. For the spin system here, the crossover procedure of the usual GA will lose important parent's 2D structure information. We will therefore introduce a modified crossover strategy for the Political Districting Problem.

Recall that the Key idea of the crossover strategy is to find the main information of the parent generation, and to help the child inherit this information. Since the aim of the political districting problem is the find some suitable districting zones, we believe the main information should be the boundary of each 


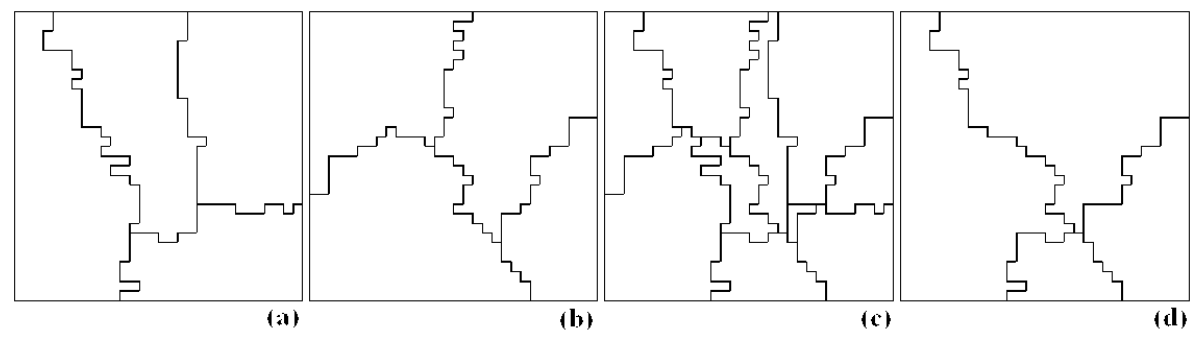

Fig. 1. A sketch of cross-over procedure. (a) The boundaries of districts from a parental chromosome; (b) Boundaries from another parental chromosome; (c) Mixed boundaries; (d) The filial chromosome.

districting zone. We thus here design a crossover process to mix the boundary of districting zone of each of the two parents, and generate a child districting zone from these mixed boundaries. Each child in some way inherit its parents' characteristic and would look similar to its parents. We illustrate the detail of our GA process in the following.

First, we should prepare a gene pool with many genes (or filial generations). To create a chromosome, we randomly put $q$ seeds with different states into an $N$ spin system and let these seeds grow until they fill all spins of system. This process will create a pool of chromosomes but only chromosomes with lower energy can be survived. These chromosomes compose of a gene pool of first generation. Once gene pool is constructed, the GA process can go on. A lot of children will be created by Cross-over and mutation procedure, and newer gene pool of children's generation will be constructed by using selection procedure.

The aim of our Crossover Strategy is to help the child generation inherit their parent's information of boundary of each districting zone. To achieve this aim, we randomly pick two chromosomes, and mix their boundaries into a new spin system and form a new chromosome. Since this chromosome with too many boundaries of districting zone, we randomly remove some boundary lines to make sure there are only $q$ different states in this chromosome. By using this procedure, the child chromosome will inherit its parents' most important information. Fig. 1 is a sketch of the cross-over procedure.

The mutation procedure of GA is to increase the diversity of the gene pool, and to avoid being trapped in a local minimum. We use a simple method to create a mutated chromosome. We pick a chromosome from gene pool, and randomly cut a small domain in the spin system and let spins to start growing diffusively from the edge of domain, till the domain is filled . By controlling the size of the cut domain, we can control the verity between mutated chromosome and original one.

From both the crossover and mutation procedures, many children chromosomes will be created. These chromosomes will go through the selection procedure to determine which chromosomes will survive. Here we use a simple rule of selection-only chromosomes with lower energy will survive. 
Table 1. A comparison of algorithms.(SR: Success Rate(\%). Total number of trials is equal to 100).

\begin{tabular}{lllllll}
\hline No. of spins & 100 & & 400 & & 900 & \\
& $E_{\text {min }}$ & $S R$ & $E_{\min }$ & $S R$ & $E_{\min }$ & $S R$ \\
\hline Method(i) & 20 & 100 & 40 & 61 & 60 & 72 \\
\hline Method(ii) & 20 & 100 & 40 & 49 & 60 & 70 \\
\hline Method(iii) & 20 & 86 & 40 & 13 & 60 & 22 \\
\hline Method(iv) & 20 & 85 & 40 & 2 & 60 & 4 \\
\hline Method(v) & 20 & - & 40 & - & 60 & - \\
\hline
\end{tabular}

\section{Result and Conclusion}

We first choose $N \times N$ square-lattice spin systems as a test of our idea. We use five methods to find the minimum of the political districting problem, namely, (i) GA with modified Crossover strategy and modified Mutation strategy, (ii) GA with modified Crossover strategy and usual single Spin-swapping Mutation strategy, (iii) GA with usual 1D Spin-swapping Crossover strategy and modified Mutation strategy, (iv) GA with usual 1D Spin-swapping Crossover strategy and single Spin-swapping Mutation strategy, and (v) simulated annealing $\operatorname{method}(\mathrm{SA})$. Table 1 is a summary of results using these five methods. In these test cases, the size of each gene-pool is equal to 400, the numbers of Crossover and Mutation in each generation are 4000 and 1000, and the total number of generation of each method is 300 . The districting parameters $\lambda_{P}$ and $\lambda_{D}$ equal to 40 and 1 . The results show that the GA with both modified Cross-over and Mutation strategy is more effective than any other GA methods.

In this paper, we mapped the Political Districting Problem onto a $q$-state Potts model in which the constraints can be written as interactions between sites or external fields acting on the system. We then show how to modify the genetic algorithm and apply it to the Political Districting Problem. Since the usual GA hardly communicate the structural information from the parent generation to their children in spin system, we design a new crossover strategy to help the child generation chromosomes inherit their parents' structural information. Our results show that this strategy work well in the Political Districting Problem.

\section{Acknowledgments}

This work was supported in part by the National Science Council, Taiwan, R.O.C. (grant no. NSC-94-2112-M-001-019 and NSC-94-2112-M-034-001).

\section{Reference}

1. Chou, C.I., Li, S.P.: Taming the Gerrymander-Statistical Physics Approach to Political Districting Problem. Physica A 369 (2006) 799-808 